\title{
Combined optical trapping and microphotoluminescence of single InP nanowires
}

\author{
Peter J. Reece, ${ }^{1, a)}$ Suriati Paiman, ${ }^{2}$ Osama Abdul-Nabi, ${ }^{1}$ Qiang Gao, ${ }^{2}$ Michael Gal, ${ }^{1}$ \\ H. Hoe Tan, ${ }^{2}$ and C. Jagadish ${ }^{2}$ \\ ${ }^{1}$ School of Physics, The University of New South Wales, Sydney, New South Wales 2052, Australia \\ ${ }^{2}$ Department of Electronic Materials Engineering, Research School of Physics and Engineering, \\ The Australian National University, Canberra, Australian Capital Territory 0200, Australia
}

(Received 1 July 2009; accepted 16 August 2009; published online 11 September 2009)

\begin{abstract}
In this letter, we demonstrate that microphotoluminescence may be combined with optical trapping for effective optical characterization of single target InP semiconductor nanowires in suspension. Using this technique, we may investigate structural properties of optically trapped nanowires, such as crystalline polytypes and stacking faults. This arrangement may also be used to resolve structural variations along the axis of the trapped nanowire. These results show that photoluminescence measurements may be coupled with optical tweezers without degrading the performance of the optical trap and provide a powerful interrogation tool for preselection of components for nanowire photonic devices. (C) 2009 American Institute of Physics. [doi:10.1063/1.3225148]
\end{abstract}

Semiconductor nanowires are likely to be a key component of any future architecture of nanophotonic and nanoelectronic integrated devices and circuits. ${ }^{1-3}$ Typically characterized by nanometer-sized features and very high aspect ratios, nanowires may be produced using a variety of fabrication techniques and can form as a complex interconnected network or as discrete elements with highly regular geometries. ${ }^{4}$ Molecular beam epitaxy (MBE) and metal organic chemical vapor deposition (MOCVD) growth techniques are particularly promising as the control of both the structural and electronic properties of semiconductor nanowires during the growth process leads to highly functional structures. $^{5}$

The potential benefits of using MOCVD/MBE grown nanowires as the basis for nanophotonic devices are numerous. The relaxation of lattice matching conditions for epitaxial growth enables nanowires to be formed on a wider range of substrates for device hybridization. Multiple axial heterostructures, such as multiquantum wells, may be formed during the growth phase $;^{6}$ these are a key element in many active devices including sources, modulators, and detectors. Similarly, electrical dopants can be introduced to create homo- and heterojunctions for electrical injection. Quantum heterostructures may be created through radial confinement (quantum wires) using core-shell nanowire structures. ${ }^{7}$

Over the past few years, there has been a growing interest in optical tweezing of high aspect ratio semiconductor nanowires and carbon nanotubes as a means of componentwise assembly of nanophotonic devices. Optical trapping of aggregates of carbon nanotubes were first seen in 2004 (Ref. 8 ) and the first individual nanowire optical micromanipulation was demonstrated in 2005 by Agarwal et al., ${ }^{9}$ who showed reorientation of II-VI compound semiconductors was possible using holographic tweezers. This work was proceeded by a survey of optical tweezing of different types of high refractive nanowires and rods ${ }^{10}$ and investigations into different trapping geometries. ${ }^{11}$ More recently, the addition of colloidal "handles" was shown to extend the trapping ca-

${ }^{a)}$ Electronic mail: p.reece@unsw.edu.au. pabilities for nanotubes. ${ }^{12}$ The first practical application of an axially trapped nonlinear potassium niobate nanocrystal has been used as a near-field scanning probe. ${ }^{13}$

In this letter, we demonstrate that microphotoluminescence $(\mu \mathrm{PL})$ may be combined with optical trapping for effective optical characterization of single target InP semiconductor nanowires in suspension. Using this technique, we may identify different crystal phases such as zinc blende (ZB) and wurtzite (WZ), as well as mixed phases. The natural alignment of the nanowires with the optical axis of the trapping beam ensures the $\mu \mathrm{PL}$ is always taken from a fixed orientation and that maximum $\mu \mathrm{PL}$ intensity is achieved without the need for active alignment. The decoupled arrangement of excitation and trapping beam enables us to probe the $\mu \mathrm{PL}$ at different points along the axis of the nanowire thereby identifying compositional variations. These results show that $\mu \mathrm{PL}$ measurements may be effectively combined with optical tweezers without degrading the performance of the optical trap and provide a powerful interrogation tool for preselection of nanowires for photonic device applications.

Figure 1(a) depicts the optical arrangement for the combined $\mu \mathrm{PL} /$ optical tweezers experiments. A linearly polarized, $1064 \mathrm{~nm}$ Ventus (Laser Quantum, U.K.) provides the trapping source; a beam expanding telescope (not shown) is used to match the beam waist to the back aperture of a 1.25 numerical aperture microscope objective (Nikon E Plan $\mathrm{x} 100$ ) and a beam steering arrangement for control of the trap position in the imaging plane. A $514.5 \mathrm{~nm}$ argon ion (Coherent I70) laser is used as the excitation source and a similar optical arrangement is used to direct the beam into the microscope. Both beams produce a diffraction limited spot in the imaging plane. Bright field imaging is used in combination with a fast frame-rate camera (Basler a602f) for recording trapped objects. $\mu \mathrm{PL}$ signal is measured using a $0.27 \mathrm{~m}$ spectrometer (SPEX) and thermoelectrically cooled charge-coupled device.

InP nanowire samples are grown by vapor-liquid-solid growth catalyzed by $30 \mathrm{~nm}$ gold nanoparticles on (111) B-oriented InP substrates using MOCVD. By controlling the 


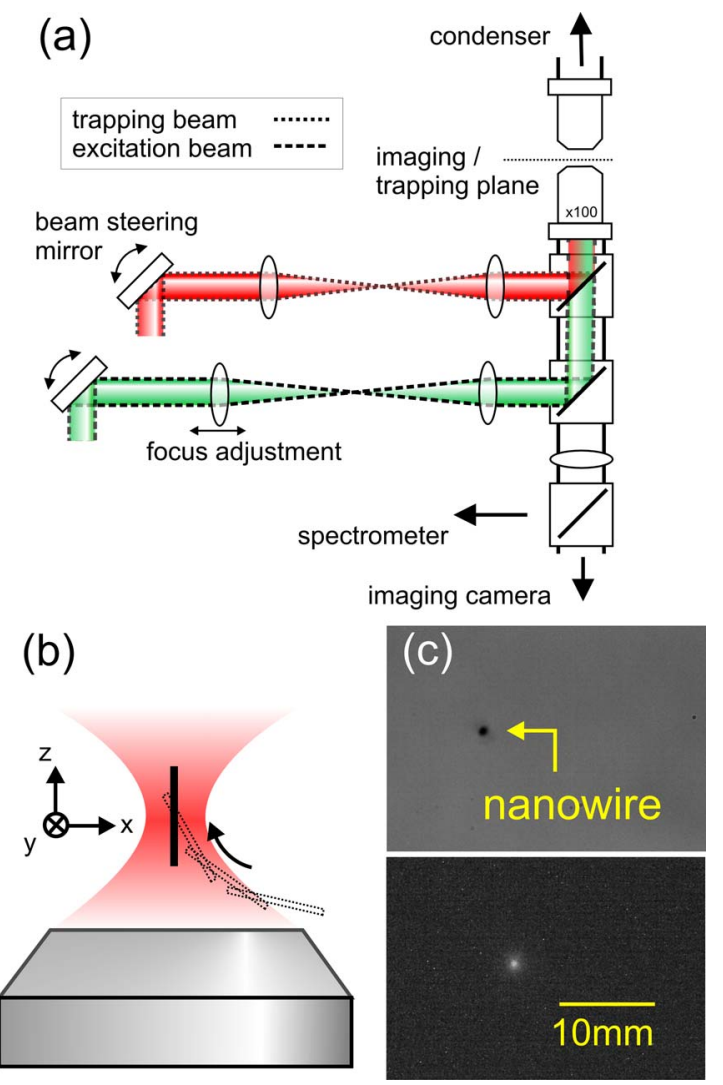

FIG. 1. (Color online) (a) Experimental set-up of the combined $\mu$ PL/optical tweezers arrangement for single nanowire optical manipulation and characterization. (b) Illustration showing the orientation of the nanowire trapped in the optical tweezers. (c) The top panel is a bright field image and bottom panel shows direct imaging of the photoluminescence when the scattered excitation beam is blocked.

growth conditions the nanowires structure may form as one of two crystalline polytypes: cubic ZB and hexagonal WZ. Single nanowires may also support mixed phases as well as structures such as ZB/WZ superlattices. The nanowires are removed from their native substrate by rubbing onto a glass slide and then a solution containing a mixture of ethanol and water (1:1) is applied to the area to create a suspension. The dimensions of the suspended nanowires are about 34-39 nm in diameter and 5-15 $\mu \mathrm{m}$ in length. Further details of the growth process may be found in Refs. 14 and 15 .

Figure 1(b) indicates the orientation of the nanowires captured in the optical tweezers; the nanowires in solution align with the long axis along the direction of propagation. The top frame of Fig. 1(c) is a bright field image of an optically trapped InP nanowire. Using a cutoff filter in front of the imaging camera to block the excitation source we are able to directly observe the near-infrared emission from the nanowire on the imaging camera; this is shown in the second frame of Fig. 1(c). The image in Fig. 2 is linked to a multimedia video of the trapped nanowire being manipulated via a steering mirror to the excitation beam site where $\mu \mathrm{PL}$ is observed. Initially the InP nanowire is below the image/ trapping plane; as the object is brought near the trap position, it is reoriented along the axis of the gradient force tweezers and held at the focus. Subsequent frames show the trapped nanowires aligned to the excitation beam using the steering mirror. Typical powers used for trapping nanowires were of the order of $20 \mathrm{~mW}$ and the power of the excitation beam

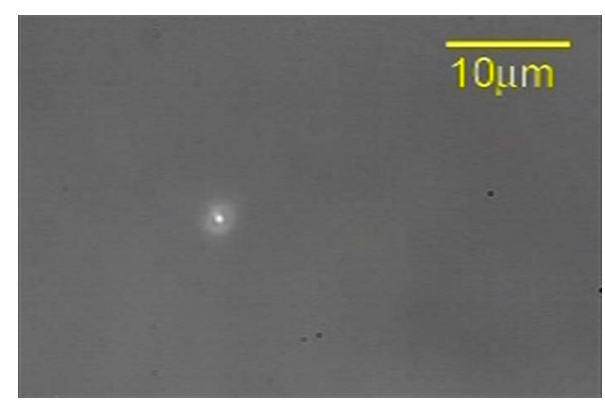

FIG. 2. (Color online) Video showing trapping and excitation of a single InP nanowire. In the video the nanowire is initially arbitrarily oriented in suspension below the trapping plane; it is then moved to the trapping site using a motorized stage where it aligns to the trapping axis. The trapped nanowire is then manipulated via the steering mirror to site of the excitation beam (enhanced online).[URL: http://dx.doi.org/10.1063/1.3225148.1]

was approximately $100 \mu \mathrm{W}$ at the focus. For excitation powers greater than the approximately $10 \%$ of the tweezers beam we find that the trapped object becomes unstable and falls out of the trap. As excitation beam powers approaching this limit it is anticipated that the stable trapping position of the nanowire will also change with respect to the tweezers beam focus, due to increase in radiation pressure.

A sequence of bright field images was taken using a fast frame rate camera and particle tracking was used to determine variations in the position of nanowires within the optical trap; the corresponding $x-y$ scatter plot of the positional fluctuations is shown in Fig. 3(a). We observe a marked asymmetry in the positional variance along the $x$-axis and $y$-axis. Strong asymmetry of the trapping potential due to the electric field's linear polarization has been observed in previous optical tweezers studies for subwavelength particle size. ${ }^{16}$ The distribution of the nanowire positions in the trap fits a Gaussian profile [Fig. 3(b)] indicating that the trapped nanowire conforms to a parabolic trapping potential. Using the equipartition method we determine the trap stiffness for the $x$-axis and $y$-axis to be 3.01 and $0.76 \mathrm{pN} / \mu \mathrm{m}$, respectively. We suggest that the asymmetry in trap stiffness observed in this case is associated with the strong polarization dependence of semiconductor nanowires; arising from large refractive index contrasts with the surrounding medium. ${ }^{17}$ We used a $1 \mu \mathrm{m}$ polymer colloidal particle to exclude the possibility of astigmatism in the optical tweezers.

Figure 4(a) shows the normalized $\mu \mathrm{PL}$ spectra of a number of individually trapped nanowires. In each case the nanowires were grown under different growth conditions to
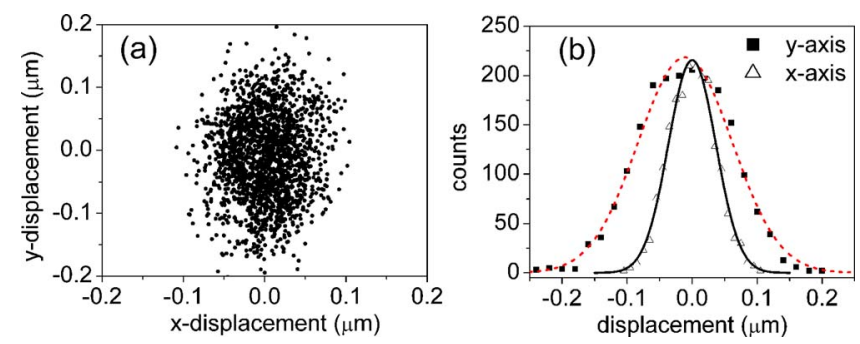

FIG. 3. (Color online) (a) Scatter diagram showing position fluctuations of the nanowire in the optical tweezers and (b) histograms showing the variations along the two major axes of the plot. The histograms have a Gaussian lineshape indicating that the optical trapping potential is approximately harmonic; an asymmetry between the $x$ - and $y$-axis is associated with the strongly polarization anisotropy of the trap. 

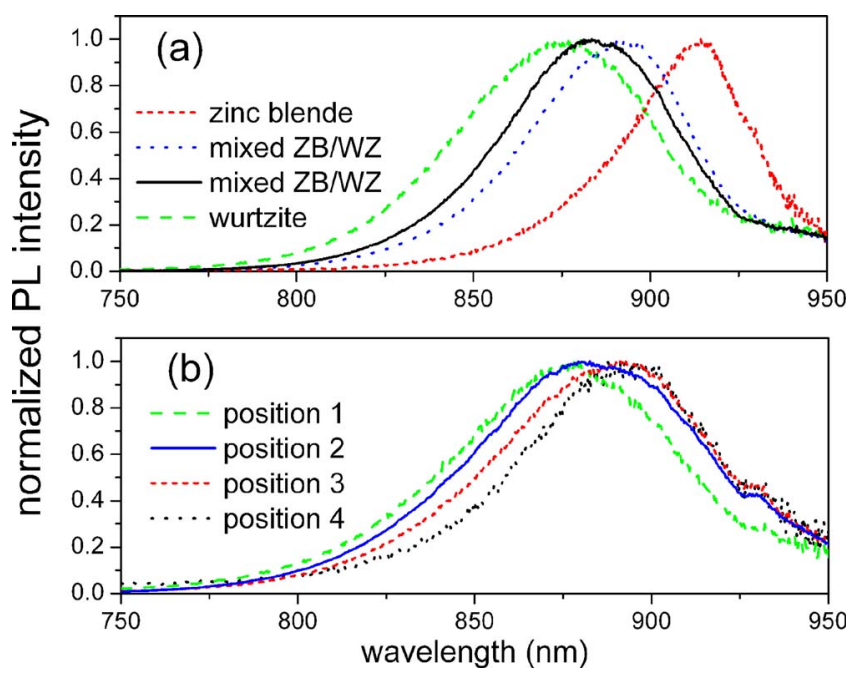

FIG. 4. (Color online) (a) Normalized $\mu \mathrm{PL}$ spectra of single optically trapped nanowires with different crystalline polytypes, i.e., ZB, WZ, and mixed WZ/ZB phases. The observed emission peaks are consistent with previous studies where PL data is correlated with the structural composition of the nanowires, as determined by TEM. (b) $\mu$ PL spectra of a single nanowire where the focus of the excitation beam is moved to different positions along the axis of the trapped nanowire. Variations in the spectra show the influence of the density of stacking faults.

achieve the desired crystal phase. The spectra include signal from predominantly ZB (peak at $918 \mathrm{~nm}$ ) and WZ (peak at $865 \mathrm{~nm}$ ) phases, as well as peaks at intermediate wavelengths corresponding to nanowires with mixed phases. The detailed crystalline structure of the nanowires examined here have been previously verified with transmission electron microscopy (TEM) and correlated with $\mu \mathrm{PL}$ measurements; for details see Ref. 14. Mixed ZB/WZ phase nanowires form as a result of the formation of stacking faults during the growth process. Stacking faults may form randomly during growth or if controlled precisely may be used to engineer homostructures such as ZB/WZ superlattices. ${ }^{15}$ These types of superlattices exhibit interesting optoelectronic characteristics due to the type-II nature of the band offsets. ${ }^{15,18}$ One of the key benefits of this technique is that if the relationship between the optical and structural properties of the nanowires are known a priori, $\mu \mathrm{PL}$ may also be used to account for variations in growth condition, which may give rise to structural variations across a wafer.

Previous polarization dependent $\mu \mathrm{PL}$ studies have shown a distinct asymmetry in PL directed parallel and perpendicular to the nanowire axis. ${ }^{19,20}$ For our particular geometry (excitation along the axis) there is no simple interpretation of the polarization dependence as it is not possible to resolve these two components. Future $\mu \mathrm{PL} /$ tweezers studies incorporating adaptive optics will allow the nanowire to be rotated into the plane where polarization dependent PL may be observed. ${ }^{9}$

As the depth of field of the microscope objective (approximately $0.5 \mu \mathrm{m}$ ) is significantly smaller than the length of the nanowires it is possible to interrogate different positions along the axis of the wire. Variations in $\mu \mathrm{PL}$ emission spectra are consistent with structural variation commonly observed along the nanowire axis, e.g., due to the density and frequency of stacking faults. As the procedure for generating the suspension results in nanowire fragments with a range of lengths, we anticipate that axial trapping position of the nanowire relative to the trapping plane will vary between objects and this will influence the region probed by the excitation beam. The excitation beam may be moved along the axis of the nanowire independently of the trapping plane by manipulating the beam expanding telescope. Figure 4(b) shows the $\mu \mathrm{PL}$ spectrum of a single nanowire at different position along the axis; in this case a monatonic shift of the emission band is observed.

In summary the ability to integrate optical tweezers with $\mu \mathrm{PL}$ enables the interrogation of optical properties of trapped nanowires prior to incorporation into an integrated nanophotonic or nanoelectronic device. This is significant as small variations in the nanowire structure can significantly affect the final electronic/optoelectronic properties of the device. The technique may be used to probe variations between different nanowires as well as structural variation along a single nanowire axis. While this study has focused specifically on InP nanowires, the approach may be universally adopted for any optically active nanowires that may be trapped using an optical tweezers arrangement.

We thank the Australian Research Council for the financial support of this research. Australian National Fabrication Facility is acknowledged for the access to the facilities used in this work.

${ }^{1}$ X. F. Duan, Y. Huang, Y. Cui, J. F. Wang, and C. M. Lieber, Nature 409, 66 (2001).

${ }^{2}$ M. S. Gudiksen, L. J. Lauhon, J. Wang, D. C. Smith, and C. M. Lieber, Nature (London) 415, 617 (2002).

${ }^{3}$ H. T. Ng, J. Li, M. K. Smith, P. Nguyen, A. Cassell, J. Han, and M. Meyyappan, Science 300, 1249 (2003).

${ }^{4}$ Y. Kim, H. J. Joyce, O. Gao, H. H. Tan, C. Jagadish, M. Paladugu, J. Zou, and A. A. Suvorova, Nano Lett. 6, 599 (2006).

${ }^{5}$ R. S. Friedman, M. C. McAlpine, D. S. Ricketts, D. Ham, and C. M. Lieber, Nature (London) 434, 1085 (2005).

${ }^{6}$ M. Paladugu, J. Zou, Y. N. Guo, X. Zhang, Y. Kim, H. J. Joyce, Q. Gao, H. H. Tan, and C. Jagadish, Appl. Phys. Lett. 93, 101911 (2008).

${ }^{7}$ L. J. Lauhon, M. S. Gudiksen, C. L. Wang, and C. M. Lieber, Nature (London) 420, 57 (2002).

${ }^{8}$ S. D. Tan, H. A. Lopez, C. W. Cai, and Y. G. Zhang, Nano Lett. 4, 1415 (2004).

${ }^{9}$ R. Agarwal, K. Ladavac, Y. Roichman, G. H. Yu, C. M. Lieber, and D. G. Grier, Opt. Express 13, 8906 (2005).

${ }^{10}$ P. J. Pauzauskie, A. Radenovic, E. Trepagnier, H. Shroff, P. D. Yang, and J. Liphardt, Nature Mater. 5, 97 (2006).

${ }^{11}$ A. van der Horst, A. I. Campbell, L. K. van Vugt, D. A. M. Vanmaekelbergh, M. Dogterom, and A. van Blaaderen, Opt. Express 15, 11629 (2007).

${ }^{12}$ J. L. Hernandez-Pozos, W. M. Lee, L. I. Vera-Robles, A. Campero, and K Dholakia, Appl. Phys. Lett. 93, 243107 (2008).

${ }^{13}$ Y. Nakayama, P. J. Pauzauskie, A. Radenovic, R. M. Onorato, R. J. Saykally, J. Liphardt, and P. D. Yang, Nature (London) 447, 1098 (2007).

${ }^{14}$ S. Paiman, Q. Gao, H. H. Tan, C. Jagadish, K. Pemasiri, M. Montazeri, H. E. Jackson, L. M. Smith, J. M. Yarrison-Rice, X. Zhang, and J. Zou, Nanotechnology 20, 225606 (2009).

${ }^{15}$ K. Pemasiri, M. Montazeri, R. Gass, L. M. Smith, H. E. Jackson, J. Yarrison-Rice, S. Paiman, Q. Gao, H. H. Tan, C. Jagadish, X. Zhang, and J. Zou, Nano Lett. 9, 648 (2009).

${ }^{16}$ A. Rohrbach, Phys. Rev. Lett. 95, 168102 (2005).

${ }^{17}$ H. E. Ruda and A. Shik, Phys. Rev. B 72, 115308 (2005).

${ }^{18}$ J. M. Bao, D. C. Bell, F. Capasso, J. B. Wagner, T. Martensson, J. Tragardh, and L. Samuelson, Nano Lett. 8, 836 (2008).

${ }^{19}$ J. F. Wang, M. S. Gudiksen, X. F. Duan, Y. Cui, and C. M. Lieber, Science 293, 1455 (2001)

${ }^{20}$ A. Mishra, L. V. Titova, T. B. Hoang, H. E. Jackson, L. M. Smith, J. M. Yarrison-Rice, Y. Kim, H. J. Joyce, Q. Gao, H. H. Tan, and C. Jagadish, Appl. Phys. Lett. 91, 263104 (2007). 